\title{
Understanding electron magnetic circular dichroism in a transition potential approach
}

\author{
J. Barthel and J. Mayer \\ Central Facility for Electron Microscopy, RWTH Aachen University, 52074 Aachen, Germany \\ and Ernst Ruska-Centre for Microscopy and Spectroscopy with Electrons, Forschungszentrum Jülich, 52425 Jülich, Germany
}

J. Rusz

Department of Physics and Materials Science, Uppsala University, Box 530, S-751 21 Uppsala, Sweden

P.-L. Ho

Beijing National Center for Electron Microscopy, Laboratory of Advanced Materials, The State Key Laboratory of New Ceramics and Fine

Processing, and School of Materials Science and Engineering, Tsinghua University, Beijing 100084, China and Central Facility for Electron Microscopy, RWTH Aachen University, 52074 Aachen, Germany

X. Y. Zhong

Beijing National Center for Electron Microscopy, Laboratory of Advanced Materials, The State Key Laboratory of New Ceramics and Fine Processing, and School of Materials Science and Engineering, Tsinghua University, Beijing 100084, China

M. Lentzen, R. E. Dunin-Borkowski, and K. W. Urban

Ernst Ruska-Centre for Microscopy and Spectroscopy with Electrons and Peter Grünberg Institute, Forschungszentrum Jülich, 52425 Jülich, Germany

H. G. Brown and S. D. Findlay

School of Physics and Astronomy, Monash University, Clayton, Victoria 3800, Australia

L. J. Allen*

School of Physics, University of Melbourne, Parkville, Victoria 3010, Australia

and Ernst Ruska-Centre for Microscopy and Spectroscopy with Electrons, Forschungszentrum Jülich, 52425 Jülich, Germany

(Received 29 November 2017; revised manuscript received 27 February 2018; published 5 April 2018)

\begin{abstract}
This paper introduces an approach based on transition potentials for inelastic scattering to understand the underlying physics of electron magnetic circular dichroism (EMCD). The transition potentials are sufficiently localized to permit atomic-scale EMCD. Two-beam and three-beam systematic row cases are discussed in detail in terms of transition potentials for conventional transmission electron microscopy, and the basic symmetries which arise in the three-beam case are confirmed experimentally. Atomic-scale EMCD in scanning transmission electron microscopy (STEM), using both a standard STEM probe and vortex beams, is discussed.
\end{abstract}

DOI: 10.1103/PhysRevB.97.144103

\section{INTRODUCTION}

Electron magnetic circular dichroism (EMCD) [1], an effect seen in electron energy-loss spectroscopy (EELS) [2], is a promising technique for studying magnetic materials in the electron microscope. In an EMCD experiment in a microscope equipped with an energy filter, the EELS spectrum probes the density of free states as a function of energy loss and momentum transfer. By comparing spectra at a given energy loss, but for a detector placed sequentially at two different symmetryrelated positions in the diffraction plane, information on the magnetic properties of the specimen can be accessed. In principle, detailed analysis of the difference spectrum yields information on the magnetic properties of the atoms in the

\footnotetext{
*1ja@unimelb.edu.au
}

specimen $[3,4]$. Since the energy of each transition depends on the bonding and also the atomic number, element-specific information is obtained. Furthermore, EMCD in the electron microscope has a higher spatial resolution and greater depth sensitivity than $\mathrm{x}$-ray magnetic circular dichroism (XMCD) [5-7]. Moreover, detecting magnetic properties with atomicsized electron probes is now feasible [8-13].

The EMCD effect is usually understood by drawing analogies with XMCD. This was first discussed in the seminal paper by Hébert and Schattschneider [14], with the first experimental confirmation of EMCD following three years later [1]. An appeal is usually made to a picture involving "virtual photons" with specific circular polarization, resulting in spectral differences which are a maximum when a virtual photon with left circular polarization and one with right circular polarization are absorbed [15]. For transition metals such as iron, cobalt, and nickel, EELS for EMCD focusses on the $L$ 
edge, corresponding to the excitation of a $2 p$ electron to an unoccupied $3 d$ state. In particular, $L_{2,3}$ EMCD in the transition metals arises primarily due to spin-orbital splitting [16] of the core level, $2 p \rightarrow 2 p_{1 / 2}$ and $2 p_{3 / 2}$, and the fact that $3 d$ valence states with spin up are preferentially occupied and have lower energy than those with spin down. The unequal occupation of spin-up and spin-down states is the origin of the magnetic properties of these metals.

In this work we adopt an approach based on transition potentials for inelastic scattering of electrons that provides a different perspective on the underlying physics of EMCD. Transition potentials are implicit in theoretical formulations of EMCD in terms of mixed dynamic form factors or density matrices, for instance, the approach described in the book by Schattschneider [17], which is also a rich source of further references, and more particularly, for example, in Refs. [1820]. Here we will explicitly consider the transition potentials themselves to provide a different perspective yielding further insight and understanding of the physics of EMCD. Consideration of the localization of the transition potentials confirms that atomic-scale EMCD is possible. The cases of two-beam and three-beam systematic rows of reflections in the diffraction plane, usually employed in EMCD experiments with conventional transmission electron microscopy (CTEM), are discussed within the transition potential framework. Fundamental symmetries seen in the formalism in the CTEM three-beam case are shown to be consistent with experimental data taken on $\mathrm{NiFe}_{2} \mathrm{O}_{4}$. We discuss the acquisition of atomic-scale EMCD in scanning transmission electron microscopy (STEM) for two different setups. First, we consider sequentially recorded images using a standard STEM probe for two symmetryrelated off-axis detector positions to obtain a difference signal. Second, we explore the use of two images using vortex probes [21,22] with opposite chirality for a detector symmetrically placed about the optical axis. In the context of EMCD, by a detector we mean an electron energy-loss spectrometer, and the "detector position" in the diffraction plane is selected by shifting the diffraction pattern with respect to the spectrometer entrance aperture. Usually only one such spectrometer is available on a microscope and the data is recorded sequentially.

\section{TRANSITION POTENTIALS}

Our starting point is the following equation for the potential describing the inelastic transition of the target electron from an occupied initial state, labeled 0 , to an unoccupied final state, labeled $n$, via a Coulomb interaction:

$$
H_{n 0}(\mathbf{r})=\frac{e^{2}}{4 \pi \varepsilon_{0}} \int u_{n}^{*}\left(\mathbf{r}^{\prime}\right) \frac{1}{\left|\mathbf{r}-\mathbf{r}^{\prime}\right|} u_{0}\left(\mathbf{r}^{\prime}\right) d \mathbf{r}^{\prime} .
$$

Let us define the interaction constant $\sigma_{n}=m /\left(2 \pi \hbar^{2} k_{n}\right)$, with $m$ the relativistically corrected mass of the incident electron, $\hbar$ the reduced Planck constant, and $k_{n}$ the wave number of the incident electron after it has excited the system to the state $n$. Then, for a suitably normalized incident plane wave, $\sigma_{n}^{2}\left|H_{n 0}(\mathbf{r})\right|^{2}$ is the probability that the inelastic transition will occur. The position vectors $\mathbf{r}$ and $\mathbf{r}^{\prime}$ are in three dimensions. The prefactor contains the magnitude of the charge of an electron $e$ and the permittivity of free space $\varepsilon_{0}$, which tacitly assumes that the Coulomb interaction is not modified by the surrounding charge distribution in the specimen. Furthermore, we consider the excitation of a single atom with the electron initially in an atomic bound state, so that the initial state in Eq. (1) can be represented by [23]

$$
u_{0}(\mathbf{r})=\frac{u_{n, l}(r)}{r} \mathcal{Y}_{j=l \pm \frac{1}{2}, m}(\theta, \phi) .
$$

The radial wave function $u_{n, l}(r)$ (real) is characterized in terms of the principal quantum number $n$ [which should not be confused with the label $n$ for a final state in Eq. (1)] and the angular momentum quantum number $l$. Taking into account spin-orbit coupling, the spin-angular functions $\mathcal{Y}_{j=l \pm \frac{1}{2}, m}(\theta, \phi)$, $\theta$ and $\phi$ polar and azimuthal angles, respectively, are defined in terms of spherical harmonics $Y_{l, m \mp \frac{1}{2}}$ as

$$
\begin{aligned}
\mathcal{Y}_{j=l \pm \frac{1}{2}, m}(\theta, \phi)= & \pm \sqrt{\frac{l \pm m+\frac{1}{2}}{2 l+1}} Y_{l, m-\frac{1}{2}}(\theta, \phi) \chi_{+} \\
& +\sqrt{\frac{l \mp m+\frac{1}{2}}{2 l+1}} Y_{l, m+\frac{1}{2}}(\theta, \phi) \chi_{-},
\end{aligned}
$$

where $m$ is the magnetic quantum number associated with the total angular momentum quantum number $j$ [16]. Generically, we denote the initial state for the bound target electron by the set of quantum numbers $\{n, l, j, m\}$. The spinors $\chi_{ \pm}$correspond to spin up (+) and spin down (-). Similarly, the final state may be expressed as

$$
u_{n}(\mathbf{r})=\frac{u_{n^{\prime}, l^{\prime}}(r)}{r} \mathcal{Y}_{j^{\prime}=l^{\prime} \pm \frac{1}{2}, m^{\prime}}(\theta, \phi)
$$

in terms of the quantum numbers $\left\{n^{\prime}, l^{\prime}, j^{\prime}, m^{\prime}\right\}$. These are atomic final states and are therefore associated with a particular atom. However, we assume that the occupancy of these final states is influenced by how that atom is bonded in the specimen.

The Fourier transform of Eq. (1) may be written as

$$
H_{n 0}(\mathbf{q})=\frac{e^{2}}{4 \pi \varepsilon_{0}} \frac{1}{\pi q^{2}} \int u_{n}^{*}(\mathbf{r}) \exp (-2 \pi i \mathbf{q} \cdot \mathbf{r}) u_{0}(\mathbf{r}) d \mathbf{r},
$$

where we have used the standard identity

$$
\int \frac{\exp (-2 \pi i \mathbf{q} \cdot \mathbf{r})}{\left|\mathbf{r}-\mathbf{r}^{\prime}\right|} d \mathbf{r}=\frac{\exp \left(-2 \pi i \mathbf{q} \cdot \mathbf{r}^{\prime}\right)}{\pi q^{2}} .
$$

To illustrate and understand the physics underlying EMCD, it will suffice to expand the exponential in Eq. (5) to first order, namely,

$$
H_{n 0}(\mathbf{q})=-\frac{e^{2}}{4 \pi \varepsilon_{0}} \frac{2 i \mathbf{q}}{q^{2}} \cdot \int u_{n}^{*}(\mathbf{r}) \mathbf{r} u_{0}(\mathbf{r}) d \mathbf{r} .
$$

This approximation [24] assumes that $\mathbf{q}$ is small and that the initial and/or final states are sufficiently localized so that the integrand is small for vectors $\mathbf{r}$ with a large magnitude. These transition potentials are only nonzero if the dipole selection rules $\Delta j= \pm 1, \Delta m=0, \pm 1$ are satisfied and the spin is conserved.

As an example, let us consider the $L_{2}$ transition from the initial state $0 \equiv\left\{2,1, \frac{1}{2}, \frac{1}{2}\right\}$ in the $2 p$ shell to the final state $n \equiv\left\{3,2, \frac{3}{2}, \frac{3}{2}\right\}$ in the $3 d$ shell. Furthermore, we assume that the electron initially has spin down and so does the final state, consistent with the dipole selection rule that this should not 
change. Then the pertinent spherical harmonics from Eq. (3) for the initial and final states, respectively, are

$$
\begin{aligned}
& Y_{1,1}(\theta, \phi)=-\frac{1}{2} \sqrt{\frac{3}{2 \pi}} \sin \theta \exp (i \phi) \text { and } \\
& Y_{2,2}(\theta, \phi)=\frac{1}{4} \sqrt{\frac{15}{2 \pi}} \sin ^{2} \theta \exp (2 i \phi),
\end{aligned}
$$

since only the spin-down terms in Eq. (3) contribute. In the same spherical coordinate system, $\mathbf{r}$ in Eq. (7) is given by

$$
\mathbf{r}=r(\sin \theta \cos \phi \hat{\mathbf{x}}+\sin \theta \sin \phi \hat{\mathbf{y}}+\cos \theta \hat{\mathbf{z}}),
$$

in terms of the usual orthonormal basis vectors for a Cartesian coordinate system. Then Eq. (7) becomes

$$
\begin{aligned}
H_{n 0}(\mathbf{q})= & \frac{e^{2}}{4 \pi \varepsilon_{0}} \frac{3 \sqrt{5}}{16 \pi} \int_{0}^{\infty} u_{3,2}(r) u_{2,1}(r) r d r \\
& \times \frac{2 i \mathbf{q}}{q^{2}} \cdot \int_{0}^{2 \pi} \int_{0}^{\pi} \sin ^{3} \theta \exp (-i \phi)(\sin \theta \cos \phi \hat{\mathbf{x}} \\
& +\sin \theta \sin \phi \hat{\mathbf{y}}+\cos \theta \hat{\mathbf{z}}) \sin \theta d \theta d \phi .
\end{aligned}
$$

Evaluating the angular integrations, we obtain

$$
\begin{aligned}
H_{n 0}^{+}(\mathbf{q})= & \frac{e^{2}}{4 \pi \varepsilon_{0}} \int_{0}^{\infty} u_{3,2}(r) u_{2,1}(r) r d r \\
& \times \frac{2 \mathbf{q}}{q^{2}} \cdot \frac{4 \sqrt{3}}{\sqrt{450}}(i \hat{\mathbf{x}}+\hat{\mathbf{y}}) \\
\equiv & F_{n 0}^{+} \frac{\mathbf{q}}{q^{2}} \cdot(i \hat{\mathbf{x}}+\hat{\mathbf{y}})=F_{n 0}^{+} \frac{i q_{x}+q_{y}}{q^{2}},
\end{aligned}
$$

where the superscript " + " has been inserted on the transition potential $H_{n 0}^{+}$and the prefactor $F_{n 0}^{+}$to explicitly denote that for this transition $\Delta m=+1$. The generic symbol $F_{n 0}^{+}$introduced here represents the general constants, the radial integration, and elements of the angular integration pertinent to a particular case. Other dipole-allowed $2 p$-to- $3 d$ transitions with $\Delta m=$ +1 yield transition potentials of a similar form to those in Eq. (11): only the constant after the dot product in the second line varies. The factor of $\sqrt{450}$ in the denominator of that constant is the lowest common multiple for all the $\Delta m=+1$ transitions, as it is for the $\Delta m=-1$ transitions, which we will consider next. The numerators can thus be used to get a measure of the relative strength of these transitions.

The transitions with $\Delta m=-1$, denoted by a superscript "-," have transition potentials of the form

$$
H_{n 0}^{-}(\mathbf{q})=F_{n 0}^{-} \frac{\mathbf{q}}{q^{2}} \cdot(i \hat{\mathbf{x}}-\hat{\mathbf{y}})=F_{n 0}^{-} \frac{i q_{x}-q_{y}}{q^{2}} .
$$

The projected transition potential $H_{n 0}\left(\mathbf{r}_{\perp}\right)$ is obtained by integrating the three-dimensional quantity in Eq. (1) over a suitable interval about the plane at $z$ as follows [25-28]:

$$
H_{n 0}\left(\mathbf{r}_{\perp}\right)=\int H_{n 0}\left(\mathbf{r}_{\perp}, z^{\prime}\right) e^{2 \pi i\left(k_{0}-k_{n}\right) z^{\prime}} d z^{\prime},
$$

where $k_{0}$ is the wave number of the incident electron, and $k_{n}$ is its wave number after the inelastic transition $\left(k_{n}<k_{0}\right)$. The projection can be carried out by taking the inverse Fourier transform of the three-dimensional potential with respect to $\mathbf{q}_{\perp}=\left(q_{x}, q_{y}, 0\right)$, the Fourier space variable conjugate to $\mathbf{r}_{\perp}$, while fixing $q_{z}=k_{0}-k_{n}$, noting that $q_{z}$ is positive and a small scattering angle approximation is implied. Using Eqs. (11) and (12) we can then write

$$
H_{n 0}^{ \pm}\left(\mathbf{r}_{\perp}\right)=2 \pi F_{n 0}^{ \pm} \hat{\mathbf{r}}_{\perp} \cdot(-\hat{\mathbf{x}} \pm i \hat{\mathbf{y}}) q_{z} K_{1}\left(2 \pi q_{z} r_{\perp}\right),
$$

where $\mathbf{r}_{\perp}=(x, y, 0)$ and we note that $\hat{\mathbf{r}}_{\perp} \cdot(-\hat{\mathbf{x}} \pm i \hat{\mathbf{y}})=$ $-\exp (\mp i \varphi)$, where $\varphi$ is the angle from the positive $x$ axis towards the $y$ axis. The function $K_{1}$ is a modified Bessel function of the second kind. A subtle point to note is that this assumes that the atom is orientated such that the direction of travel of the incoming beam of electrons coincides with the axis with respect to which the magnetic quantum number is defined. We will not elaborate on this further here, except to note that this is pertinent when detecting in-plane magnetization [29]. For the details of how this Fourier transform can be done, we refer the reader to Eq. (14) in Ref. [30], where the substitution $\varepsilon_{\perp}^{\kappa}=i \hat{\mathbf{x}} \pm \hat{\mathbf{y}}$ in that equation yields the required result. In a similar way we can show that the transition potentials for transitions with $\Delta m=0$ are of the form

$$
H_{n 0}^{0}(\mathbf{q})=F_{n 0}^{0} \frac{q_{z}}{q^{2}},
$$

so that in real space

$$
H_{n 0}^{0}\left(\mathbf{r}_{\perp}\right)=2 \pi F_{n 0}^{0} q_{z} K_{0}\left(2 \pi q_{z} r_{\perp}\right),
$$

where $K_{0}$ is a modified Bessel function of the second kind.

Figure 1(a) shows the amplitude and the phases of $\hat{\mathbf{r}}_{\perp} \cdot(-\hat{\mathbf{x}} \pm$ $i \hat{\mathbf{y}}) q_{z} K_{1}\left(2 \pi q_{z} r_{\perp}\right)$ corresponding to $\Delta m= \pm 1$. In each case, the phase is consistent with there being a vortex structure but with differing chirality. Also shown is $q_{z} K_{0}\left(2 \pi q_{z} r_{\perp}\right)$, and in that case the phase is a constant. The amplitudes have been scaled in the ratio that they contribute as outlined in the discussion of Fig. 2 in Sec. III. It is clear from the amplitude that the localization of the projected transition potentials in real space is determined by $q_{z} \approx k_{0} E_{\text {loss }} / 2 E_{0}$, where $E_{0}$ denotes the energy of the incident electron and $E_{\text {loss }}$ its energy loss [31]. We have assumed an energy loss of $715 \mathrm{eV}$ (the approximate energy loss for the $\mathrm{Fe} L_{2,3}$ inelastic transition) and an incident electron energy of $200 \mathrm{keV}\left(q_{z} \approx\right.$ $40 \AA^{-1} \times 0.715 \mathrm{keV} / 400 \mathrm{keV} \approx 0.07 \AA^{-1}$ ) in calculating the amplitudes in Fig. 1(a). The diameter $d_{50}=3.6 \AA$ marked in Fig. 1(a) contains $50 \%$ of the total potential amplitude $\left|H_{n 0}^{ \pm}\left(\mathbf{r}_{\perp}\right)\right|$, evaluated by integrating in two dimensions. In Fig. 1(b), $d_{50}$ and $d_{95}$ are plotted for a range of energy losses and incident electron energies relevant in CTEM and STEM EELS. As expected, the larger the energy loss, the more localized is the transition potential in real space. Since $q_{z}$ is inversely proportional to $E_{0}$, we also see increasing localization with decreasing incident electron energy for a fixed energy loss. The transition amplitudes $\left|H_{n 0}^{0}\left(\mathbf{r}_{\perp}\right)\right|$ actually have slightly larger values of $d_{50}$ and $d_{95}$, but in what follows the $\left|H_{n 0}^{ \pm}\left(\mathbf{r}_{\perp}\right)\right|$ contributions will be more important. The results shown in Fig. 1(b), especially those for a diameter $d_{50}$, are consistent with atomic-scale information being accessible using EMCD.

\section{THE PHYSICS OF THE EMCD EFFECT}

We start from a fundamental equation for an inelastic scattering event from an initial state 0 to a final state $n$ occurring 

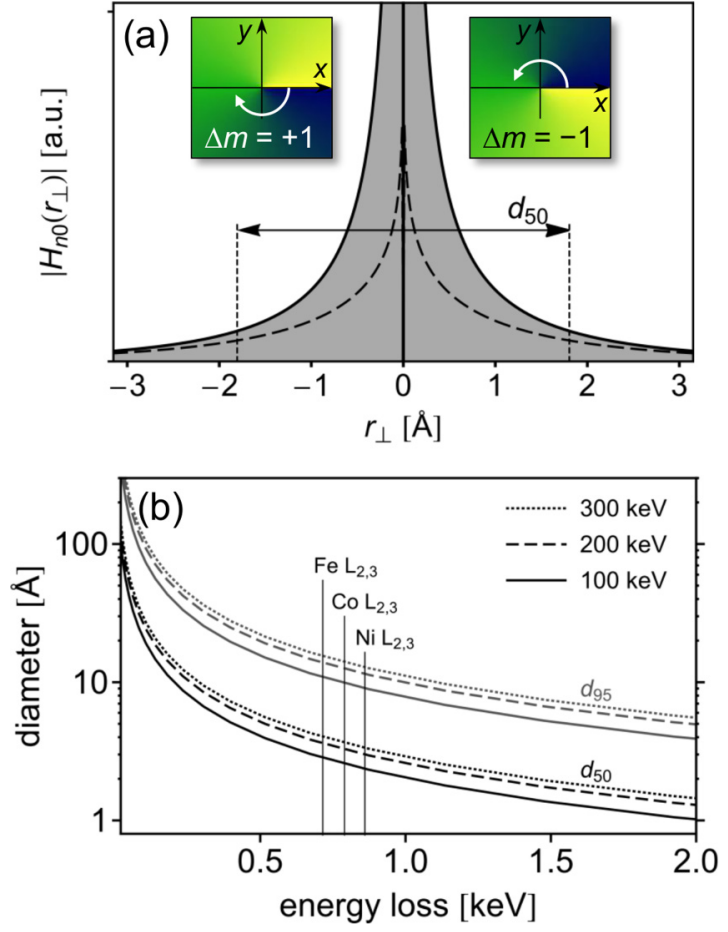

FIG. 1. (a) The common amplitude (solid curve) and the phase maps (inset) of the transition potentials $H_{n 0}^{ \pm}\left(\mathbf{r}_{\perp}\right)$ showing the localization as well as the vortex structure in their phases. The reversal of the azimuthal phase ramp in the insets, which increases in the direction of the arrows, reflects the different sign of the topological charge (chirality) occurring in the respective transitions with $\Delta m=+1$ and $\Delta m=-1$. Also shown (dashed curve) is $H_{n 0}^{0}(\Delta m=0)$, scaled as discussed in the text, and for which the phase is a constant. The amplitudes in (a) correspond to an $\mathrm{Fe} L_{2,3}$ inelastic transition with an energy loss around $715 \mathrm{eV}$ and for an incident electron energy of $200 \mathrm{keV}$. The double-headed arrow indicates the diameter $d_{50}=3.6$ $\AA$ containing $50 \%$ of the total potential amplitude for the $H_{n 0}^{ \pm}\left(\mathbf{r}_{\perp}\right)$ cases. (b) The diameters $d_{50}$ and $d_{95}$ are plotted for different incident electron energies and for a range of energy losses relevant in CTEM and STEM EELS experiments.

at a depth $z$ in a specimen [25-28]:

$$
\psi_{n}\left(\mathbf{r}_{\perp}, z\right)=-i \sigma_{n} H_{n 0}\left(\mathbf{r}_{\perp}\right) \psi_{0}\left(\mathbf{r}_{\perp}, z\right) .
$$

Here $\psi_{0}\left(\mathbf{r}_{\perp}, z\right)$ is the wave function of the incident probe electron before the inelastic transition and $\psi_{n}\left(\mathbf{r}_{\perp}, z\right)$ describes this electron after the inelastic scattering event. The interaction constant $\sigma_{n}$ in Eq. (17) was introduced after Eq. (1).

Using the convolution theorem, the two-dimensional Fourier transform of Eq. (17) can be written as

$$
\psi_{n}\left(\mathbf{q}_{\perp}, z\right)=-i \sigma_{n} \int H_{n 0}\left(\mathbf{q}_{\perp}^{\prime}\right) \psi_{0}\left(\mathbf{q}_{\perp}-\mathbf{q}_{\perp}^{\prime}, z\right) d \mathbf{q}_{\perp}^{\prime} .
$$

The contribution to the signal from the inelastic transition $0 \rightarrow$ $n$ at a point $\mathbf{q}_{\perp}$ in the diffraction plane can then be expressed as

$$
\begin{aligned}
\left|\psi_{n}\left(\mathbf{q}_{\perp}, z\right)\right|^{2}= & \sigma_{n}^{2} \iint H_{n 0}^{*}\left(\mathbf{q}_{\perp}^{\prime}\right) H_{n 0}\left(\mathbf{q}_{\perp}^{\prime \prime}\right) \\
& \times \psi_{0}^{*}\left(\mathbf{q}_{\perp}-\mathbf{q}_{\perp}^{\prime}, z\right) \psi_{0}\left(\mathbf{q}_{\perp}-\mathbf{q}_{\perp}^{\prime \prime}, z\right) d \mathbf{q}_{\perp}^{\prime} d \mathbf{q}_{\perp}^{\prime \prime}
\end{aligned}
$$

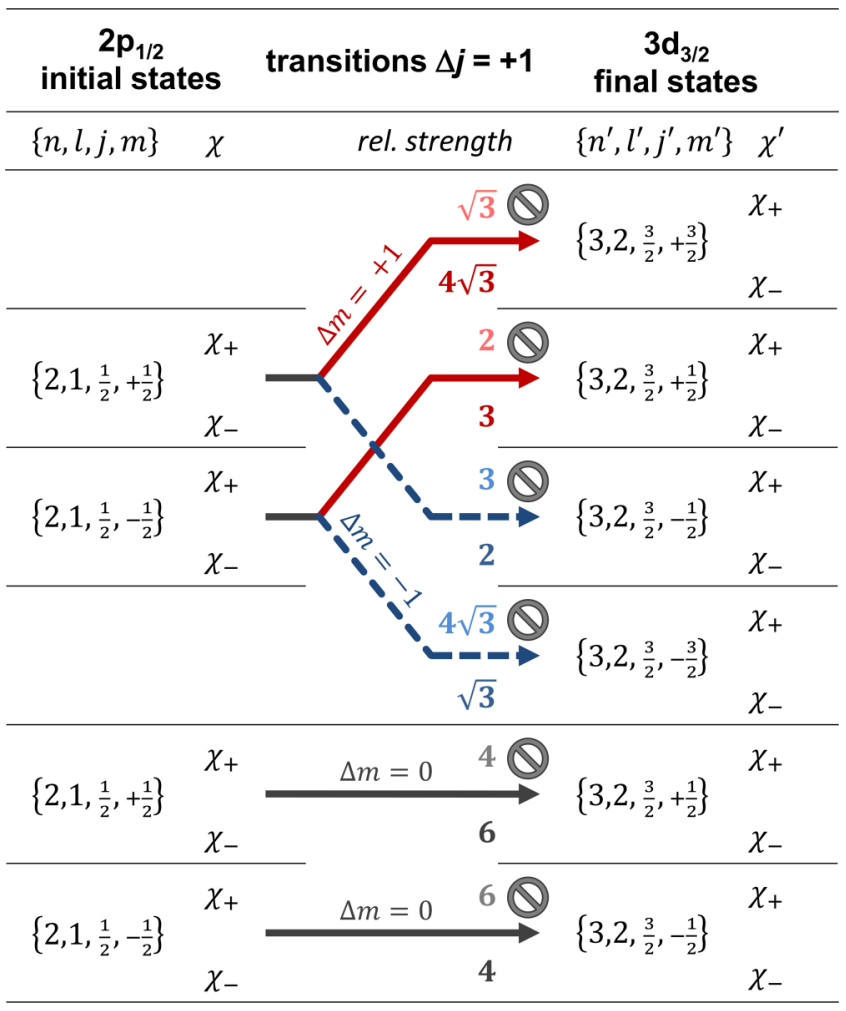

FIG. 2. Schematic $L_{2}$ transition scheme illustrating dipoleallowed transitions with $\Delta m=+1$ (red solid lines), $\Delta m=-1$ (blue dashed lines), and $\Delta m=0$ (solid black lines). $L_{2}$ transitions blocked by occupation of final states assuming a fully spin-up polarized material, denoted by the "prohibited" signs, lead to an imbalance in the strength of transitions with $\Delta m=+1$ (stronger) compared to those with $\Delta m=-1$ (weaker). The relative strengths shown refer to the amplitude of each transition, as discussed in the paragraph after that containing Eq. (11).

$$
\begin{aligned}
\approx & \sigma_{n}^{2} \sum_{\mathbf{q}_{\perp}^{\prime}, \mathbf{q}_{\perp}^{\prime \prime}} H_{n 0}^{*}\left(\mathbf{q}_{\perp}^{\prime}\right) H_{n 0}\left(\mathbf{q}_{\perp}^{\prime \prime}\right) \\
& \times \psi_{0}^{*}\left(\mathbf{q}_{\perp}-\mathbf{q}_{\perp}^{\prime}, z\right) \psi_{0}\left(\mathbf{q}_{\perp}-\mathbf{q}_{\perp}^{\prime \prime}, z\right),
\end{aligned}
$$

where the integrations have been written in a discretized form. This assumes that the outgoing waves after inelastic scattering do not channel in the specimen. For the case of transitions with $\Delta m= \pm 1$, those pertinent to EMCD, the products of transition potentials that occur in the previous equation can be written, using Eqs. (11) and (12), as

$$
\begin{aligned}
& H_{n 0}^{ \pm *}\left(\mathbf{q}_{\perp}^{\prime}\right) H_{n 0}^{ \pm}\left(\mathbf{q}_{\perp}^{\prime \prime}\right) \\
& \quad=\left[F_{n 0}^{ \pm}\right]^{2}\left[\frac{\mathbf{q}_{\perp}^{\prime} \cdot \mathbf{q}_{\perp}^{\prime \prime}}{q^{\prime 2} q^{\prime \prime 2}} \mp i \frac{\left(\mathbf{q}_{\perp}^{\prime} \times \mathbf{q}_{\perp}^{\prime \prime}\right) \cdot \hat{\mathbf{z}}}{q^{\prime 2} q^{\prime \prime 2}}\right] \\
& \quad \equiv A_{n 0}^{ \pm}\left(\mathbf{q}_{\perp}^{\prime}, \mathbf{q}_{\perp}^{\prime \prime}\right) \mp i B_{n 0}^{ \pm}\left(\mathbf{q}_{\perp}^{\prime}, \mathbf{q}_{\perp}^{\prime \prime}\right) .
\end{aligned}
$$

The first term $A_{n 0}^{ \pm}$in the large square brackets in the second line of Eq. (20) is symmetric with respect to interchange of $\mathbf{q}_{\perp}^{\prime}$ and $\mathbf{q}_{\perp}^{\prime \prime}$ and is the same for transitions with $\Delta m= \pm 1$. However the second term $B_{n 0}^{ \pm}$in the same square brackets, containing the cross product, is antisymmetric with respect to the interchange of $\mathbf{q}_{\perp}^{\prime}$ and $\mathbf{q}_{\perp}^{\prime \prime}$ and is of opposite sign for the $\Delta m=+1$ and $\Delta m=-1$ cases. We have chosen to indicate 
the arguments of $A_{n 0}^{ \pm}$and $B_{n 0}^{ \pm}$as $\mathbf{q}_{\perp}^{\prime}$ and $\mathbf{q}_{\perp}^{\prime \prime}$, but we emphasize that the scalar quantities $q^{\prime}$ and $q^{\prime \prime}$ still contain a $q_{z}$ component. We also note that such products of transition potentials can be related to the mixed dynamic form factor that occurs in inelastic scattering $[17,28,32]$, a quantity which has been used in other work on EMCD [1,14,17,33,34]. Note the formal similarity with Eq. (12) in Ref. [34].

If the sum over contributions from all transitions with $\Delta m=+1$ is the same as that from transitions with $\Delta m=-1$, for a given atom, then the contributions from the antisymmetric terms will cancel. However, in a magnetic atom where a fraction of the $3 d$ states is already filled with electrons with spin up, this will not be the case. For simplicity, let us assume that half of the $3 d$ states are filled with electrons with spin up. Then, consistent with the dipole selection rule that spin should not change in a transition, only transitions from initial states with spin down to final states with spin down can occur. This means that the $L_{2}$ transition $0 \equiv\left\{2,1, \frac{1}{2}, \frac{1}{2}\right\} \rightarrow$ $n \equiv\left\{3,2, \frac{3}{2}, \frac{3}{2}\right\}$ explicitly evaluated in Sec. II is an example of an allowed transition. Initial and final states with spin down imply that in calculating a transition the first term on the right-hand side of Eq. (3) does not contribute in each case. This has the consequence that the contribution from $\Delta m= \pm 1$ transitions will be of a different strength, as illustrated in Fig. 2. The relative transition strengths shown are calculated by performing the spin-angular part of the integration implicit in Eq. (7). In Eq. (11) this is the factor $4 \sqrt{3}$. Angular components of the initial and final states are calculated using Eq. (3) while taking into account which spin states may contribute, i.e., only spin-down states in our example. In this scenario transition probabilities for allowed $L_{2}$ transitions with $\Delta m=+1$ are 8.1 times larger than those with $\Delta m=-1$, as calculated by summing up the squared strengths of the allowed transitions in Fig. 2. Similarly, once again assuming that all $3 d$ states with spin up are filled and all those with spin down unfilled, we can calculate the ratio of transition probabilities for $L_{3}$ transitions with $\Delta m=+1$ to those with $\Delta m=-1$ as 0.6 . While a more elaborate model may be needed to calculate these ratios more accurately, the fact that the first ratio is greater than unity and the second less than unity is consistent with the opposite sign of the EMCD effect observed in the experiment for $L_{2}$ and $L_{3}$ transitions. These considerations are the essence of the EMCD effect.

Measuring the energy-loss signal at two points in the diffraction plane, where first $\mathbf{q}_{\perp}^{\prime} \times \mathbf{q}_{\perp}^{\prime \prime}$ occurs in Eq. (20) and then second $\mathbf{q}_{\perp}^{\prime \prime} \times \mathbf{q}_{\perp}^{\prime}$, and subtracting these signals then leads to a difference signal which indicates the presence of magnetization and in principle allows one to determine its magnitude. We will discuss this more explicitly in the next section. In STEM one can also exploit this asymmetry by scanning the probe, as will be discussed in Sec. V.

\section{ELECTRON MAGNETIC CIRCULAR DICHROISM IN CONVENTIONAL TRANSMISSION ELECTRON MICROSCOPY}

Let us now assume that we are in the context of conventional transmission electron microscopy with plane-wave illumination. It is then convenient to write the probe wave function $\psi_{0}\left(\mathbf{r}_{\perp}, z\right)$ in the crystal in terms of Bloch states as [35]

$$
\psi_{0}\left(\mathbf{r}_{\perp}, z\right)=\sum_{\mathbf{G}} \sum_{j} \alpha^{j} C_{\mathbf{G}}^{j} \exp \left(2 \pi i \gamma^{j} z\right) \exp \left(2 \pi i \mathbf{G} \cdot \mathbf{r}_{\perp}\right),
$$

where, without loss of generality, an overall phase factor $\exp (2 \pi i K z)$, with $K$ the wave number of the incident electron corrected for refraction, has been ignored. The $\mathbf{G}$ are reciprocal lattice vectors, $\alpha^{j}$ is the amplitude of Bloch state $j, C_{\mathbf{G}}^{j}$ are components of the eigenvector for state $j$, and $\gamma^{j}$ are related to the eigenvalues. Defining

$$
C_{\mathbf{G}}(z)=\sum_{j} \alpha^{j} C_{\mathbf{G}}^{j} \exp \left(2 \pi i \gamma^{j} z\right),
$$

we can write

$$
\psi_{0}\left(\mathbf{r}_{\perp}, z\right)=\sum_{\mathbf{G}} C_{\mathbf{G}}(z) \exp \left(2 \pi i \mathbf{G} \cdot \mathbf{r}_{\perp}\right) .
$$

From this Fourier expansion it is clear that the Fourier coefficients are only nonzero at the physical reciprocal lattice points $\mathbf{G}$. Therefore the arguments in the probe wave function in Eq. (19) should be written as

$$
\mathbf{q}_{\perp}-\mathbf{q}_{\perp}^{\prime}=\mathbf{G}, \quad \mathbf{q}_{\perp}-\mathbf{q}_{\perp}^{\prime \prime}=\mathbf{H}
$$

and the discretized form of Eq. (19) reduces to

$$
\begin{aligned}
\left|\psi_{n}\left(\mathbf{q}_{\perp}, z\right)\right|^{2}= & \sigma_{n}^{2} \sum_{\mathbf{G}, \mathbf{H}} H_{n 0}^{*}\left(\mathbf{q}_{\perp}-\mathbf{G}\right) H_{n 0}\left(\mathbf{q}_{\perp}-\mathbf{H}\right) \\
& \times\left|C_{\mathbf{G}}(z)\right|\left|C_{\mathbf{H}}(z)\right| \exp \left\{i\left[\phi_{\mathbf{H}}(z)-\phi_{\mathbf{G}}(z)\right]\right\},
\end{aligned}
$$

where $\phi_{\mathbf{G}}(z)$ and $\phi_{\mathbf{H}}(z)$ are the phases of the Fourier coefficients $C_{\mathbf{G}}(z)$ and $C_{\mathbf{H}}(z)$ given by Eq. (22). This can now be written in the form

$$
\begin{aligned}
\left|\psi_{n}\left(\mathbf{q}_{\perp}, z\right)\right|^{2}= & \sigma_{n}^{2} \sum_{\mathbf{G}}\left|C_{\mathbf{G}}\right|^{2}\left|H_{n 0}\left(\mathbf{q}_{\perp}-\mathbf{G}\right)\right|^{2} \\
& +\sigma_{n}^{2} \sum_{\mathbf{G} \neq \mathbf{H}}\left|C_{\mathbf{G}}(z)\right|\left|C_{\mathbf{H}}(z)\right| \Re\left\{\operatorname { e x p } \left[i \left(\phi_{\mathbf{H}}(z)\right.\right.\right. \\
& \left.\left.\left.-\phi_{\mathbf{G}}(z)\right)\right] H_{n 0}^{*}\left(\mathbf{q}_{\perp}-\mathbf{G}\right) H_{n 0}\left(\mathbf{q}_{\perp}-\mathbf{H}\right)\right\},
\end{aligned}
$$

where the first term on the right-hand side contains the diagonal terms in the summation in Eq. (25). The $\Re$ denotes the real part of the quantity in curly brackets and is obtained by adding "off-diagonal" complex conjugate quantities in Eq. (25).

\section{A. Two-beam case}

If we now consider a simple two-beam case ( $\mathbf{0}$ and $\mathbf{G})$, conditions that can be realized experimentally to a good approximation, then Eq. (26) reduces to

$$
\begin{aligned}
\left|\psi_{n}\left(\mathbf{q}_{\perp}, z\right)\right|^{2}= & \sigma_{n}^{2}\left[\left|C_{\mathbf{0}}(z)\right|^{2}\left|H_{n 0}\left(\mathbf{q}_{\perp}\right)\right|^{2}+\left|C_{\mathbf{G}}\right|^{2}\left|H_{n 0}\left(\mathbf{q}_{\perp}-\mathbf{G}\right)\right|^{2}\right] \\
& +2 \sigma_{n}^{2}\left|C_{\mathbf{0}}(z)\right|\left|C_{\mathbf{G}}(z)\right| \Re\left\{\exp \left[i\left(\phi_{\mathbf{G}}(z)-\phi_{\mathbf{0}}(z)\right)\right]\right. \\
& \left.\times H_{n 0}^{*}\left(\mathbf{q}_{\perp}\right) H_{n 0}\left(\mathbf{q}_{\perp}-\mathbf{G}\right)\right\} .
\end{aligned}
$$

Referring once again specifically to transitions with $\Delta m=$ \pm 1 , these will contribute to the signal at a point $\mathbf{q}_{\perp}$ via factors in the second summation in Eq. (26) of the form 


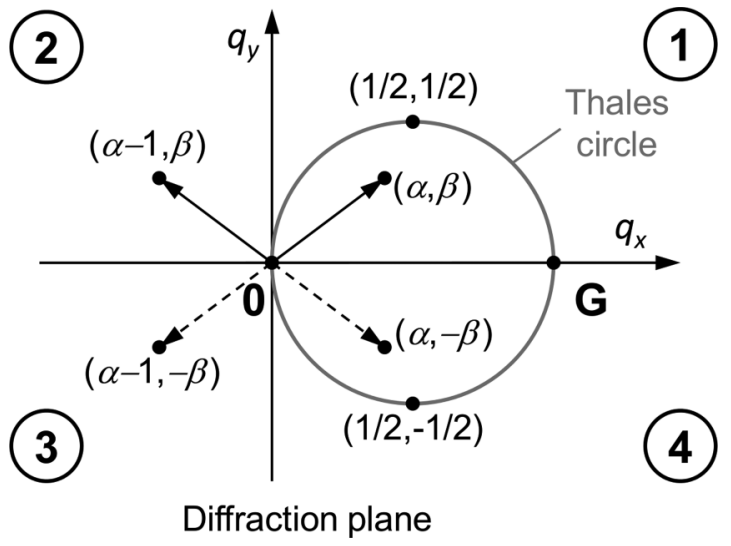

FIG. 3. Two-beam conditions for CTEM showing the various vectors in the upper half-plane (solid lines) and lower half-plane (dashed lines) that are pertinent to Eqs. (29)-(31). The Thales circle is the locus of all points for which $(\alpha, \beta)$ and $(\alpha-1, \beta)$ are orthogonal.

$\Re\left[\exp (i \phi) H_{n 0}^{ \pm *}\left(\mathbf{q}_{\perp}^{\prime}\right) H_{n 0}^{ \pm}\left(\mathbf{q}_{\perp}^{\prime \prime}\right)\right]$, where $\phi=\phi_{\mathbf{H}}(z)-\phi_{\mathbf{G}}(z)$ is a phase difference which varies as a function of depth $z$. Using Eq. (20), these factors can be written in the form

$$
\begin{aligned}
\Re & {\left[\exp (i \phi) H_{n 0}^{ \pm *}\left(\mathbf{q}_{\perp}^{\prime}\right) H_{n 0}^{ \pm}\left(\mathbf{q}_{\perp}^{\prime \prime}\right)\right] } \\
& =\left[F_{n 0}^{ \pm}\right]^{2}\left[\cos \phi \frac{\mathbf{q}_{\perp}^{\prime} \cdot \mathbf{q}_{\perp}^{\prime \prime}}{q^{\prime 2} q^{\prime \prime 2}} \pm \sin \phi \frac{\left(\mathbf{q}_{\perp}^{\prime} \times \mathbf{q}_{\perp}^{\prime \prime}\right) \cdot \hat{\mathbf{z}}}{q^{\prime 2} q^{\prime 2}}\right] \\
& =\cos \phi A_{n 0}^{ \pm} \pm \sin \phi B_{n 0}^{ \pm} .
\end{aligned}
$$

For the two-beam case, let us consider a point $\mathbf{q}_{\perp}$ in the diffraction plane in the first quadrant and express this in units of the magnitude of $\mathbf{G}$ in the short-hand notation $\mathbf{q}_{\perp} \equiv(\alpha, \beta)$, as indicated in Fig. 3. The Thales circle $[1,15]$ shown in Fig. 3 is the locus of all points for which $(\alpha, \beta)$ and $(\alpha-1, \beta)$ are orthogonal. At the points $(1 / 2, \pm 1 / 2)$ indicated, these vectors have equal magnitude and are optimal choices for measuring the EMCD difference signal.

Let us consider the "off-diagonal" contributions in Eq. (27) from the transitions with $\Delta m= \pm 1$. Summing these contributions and assuming $\mathbf{G}$ is on the positive $q_{x}$ axis, we may write

$$
\begin{aligned}
\sum_{n} \mid & \left.\psi_{n}^{+}(\alpha, \beta)\right|^{2}+\sum_{n^{\prime}}\left|\psi_{n^{\prime}}^{-}(\alpha, \beta)\right|^{2} \\
= & 2\left|C_{\mathbf{0}}\right|\left|C_{\mathbf{G}}\right| \sum_{n} \sigma_{n}^{2}\left[\cos \phi A_{n 0}^{+}((\alpha, \beta),(\alpha-1, \beta))\right. \\
& \left.+\sin \phi B_{n 0}^{+}((\alpha, \beta),(\alpha-1, \beta))\right] \\
& +2\left|C_{\mathbf{0}}\right|\left|C_{\mathbf{G}}\right| \sum_{n^{\prime}} \sigma_{n^{\prime}}^{2}\left[\cos \phi A_{n^{\prime} 0}^{-}((\alpha, \beta),(\alpha-1, \beta))\right. \\
& \left.-\sin \phi B_{n^{\prime} 0}^{-}((\alpha, \beta),(\alpha-1, \beta))\right],
\end{aligned}
$$

where the dependence on $z$ has been suppressed for notational clarity and $\phi \equiv \phi_{\mathbf{G}}(z)-\phi_{\mathbf{0}}(z)$. The summation over $n$ is for transitions with $\Delta m=+1$ and that over $n^{\prime}$ is for transitions with $\Delta m=-1$. For the point $\mathbf{q}_{\perp}$ in the fourth quadrant that is mirrored across the $q_{x}$ axis $(\beta \rightarrow-\beta)$ we can write

$$
\begin{aligned}
& \sum_{n}\left|\psi_{n}^{+}(\alpha,-\beta)\right|^{2}+\sum_{n^{\prime}}\left|\psi_{n^{\prime}}^{-}(\alpha,-\beta)\right|^{2} \\
& =2\left|C_{\mathbf{0}}\right|\left|C_{\mathbf{G}}\right| \sum_{n} \sigma_{n}^{2}\left[\cos \phi A_{n 0}^{+}((\alpha,-\beta),(\alpha-1,-\beta))\right. \\
& \left.\quad+\sin \phi B_{n 0}^{+}((\alpha,-\beta),(\alpha-1,-\beta))\right] \\
& \quad+2\left|C_{\mathbf{0}}\right|\left|C_{\mathbf{G}}\right| \sum_{n^{\prime}} \sigma_{n^{\prime}}^{2}\left[\cos \phi A_{n^{\prime} 0}^{-}((\alpha,-\beta),(\alpha-1,-\beta))\right. \\
& \left.\quad-\sin \phi B_{n^{\prime} 0}^{-}((\alpha,-\beta),(\alpha-1,-\beta))\right] .
\end{aligned}
$$

Inspection of the dot and cross products of the pairs of vectors occurring in Eqs. (29) and (30), using Fig. 3, reveals that the cross products are of opposite sign, and so if we subtract Eq. (30) from Eq. (29) we are left with

$$
\begin{aligned}
\sum_{n} & \left|\psi_{n}^{+}(\alpha, \beta)\right|^{2}+\sum_{n^{\prime}}\left|\psi_{n^{\prime}}^{-}(\alpha, \beta)\right|^{2} \\
& -\sum_{n}\left|\psi_{n}^{+}(\alpha,-\beta)\right|^{2}-\sum_{n^{\prime}}\left|\psi_{n^{\prime}}^{-}(\alpha,-\beta)\right|^{2} \\
= & 4\left|C_{\mathbf{0}}\right|\left|C_{\mathbf{G}}\right| \sin \phi \sum_{n} \sigma_{n}^{2} B_{n 0}^{+}((\alpha, \beta),(\alpha-1, \beta)) \\
& -4\left|C_{\mathbf{0}}\right|\left|C_{\mathbf{G}}\right| \sin \phi \sum_{n^{\prime}} \sigma_{n^{\prime}}^{2} B_{n^{\prime} 0}^{-}((\alpha, \beta),(\alpha-1, \beta)) .
\end{aligned}
$$

If we had carried along the "diagonal" terms in Eq. (27) in the derivation, they would cancel at this point. Clearly, if the transitions $\Delta m= \pm 1$ are of equal strength, then $\sum_{n} \sigma_{n}^{2} B_{n 0}^{+}=$ $\sum_{n^{\prime}} \sigma_{n^{\prime}}^{2} B_{n^{\prime} 0}^{-}$and there is no difference signal. However, as we have seen in the previous section, for atoms with a magnetic moment this is not the case, and the terms on the right-hand side of Eq. (31) do not cancel. It is clear that this difference signal will be a maximum when $\phi=\phi_{\mathbf{G}}-\phi_{\mathbf{0}}=\left(N+\frac{1}{2}\right) \pi, N$ an integer. We also note that since the total signal is obtained by integrating Eq. (31) over all the thickness values $z$ implicit in that equation, the overall thickness of the specimen plays a role in maximizing the EMCD signal $[13,15,33,36,37]$. The results for the two-beam case hold for any pair of points $(\alpha, \beta)$ and $(\alpha,-\beta)$ and not just the points $(1 / 2,1 / 2)$ and $(1 / 2,-1 / 2)$ on the Thales circle (the ideal case).

\section{B. Three-beam systematic row case}

Now let us consider the three-beam systematic row of reflections $-\mathbf{G}, \mathbf{0}$, and $\mathbf{G}$, also attainable to a reasonable approximation experimentally by judicious tilting of the specimen. Figure 4 shows an experimental diffraction pattern for $300-\mathrm{keV}$ incident electrons for the relevant beams in a (400) systematic row obtained by tilting the sample $9^{\circ}$ away from the [001] zone axis towards [010] in $\mathrm{NiFe}_{2} \mathrm{O}_{4}$, which is centrosymmetric. The red circles in Fig. 4 indicate detector apertures placed symmetrically in quadrants $1-4$ in the diffraction plane, to be referred to later.

From Eq. (26), the contribution to the intensity measured at the point $\mathbf{q}_{\perp}$ in the diffraction plane from one of the possible transitions is given by

$$
\begin{aligned}
\left|\psi_{n}\left(\mathbf{q}_{\perp}\right)\right|^{2}= & \sigma_{n}^{2}\left[\left|C_{-\mathbf{G}}\right|^{2}\left|H_{n 0}\left(\mathbf{q}_{\perp}+\mathbf{G}\right)\right|^{2}\right. \\
& \left.+\left|C_{\mathbf{0}}\right|^{2}\left|H_{n 0}\left(\mathbf{q}_{\perp}\right)\right|^{2}+\left|C_{\mathbf{G}}\right|^{2}\left|H_{n 0}\left(\mathbf{q}_{\perp}-\mathbf{G}\right)\right|^{2}\right]
\end{aligned}
$$




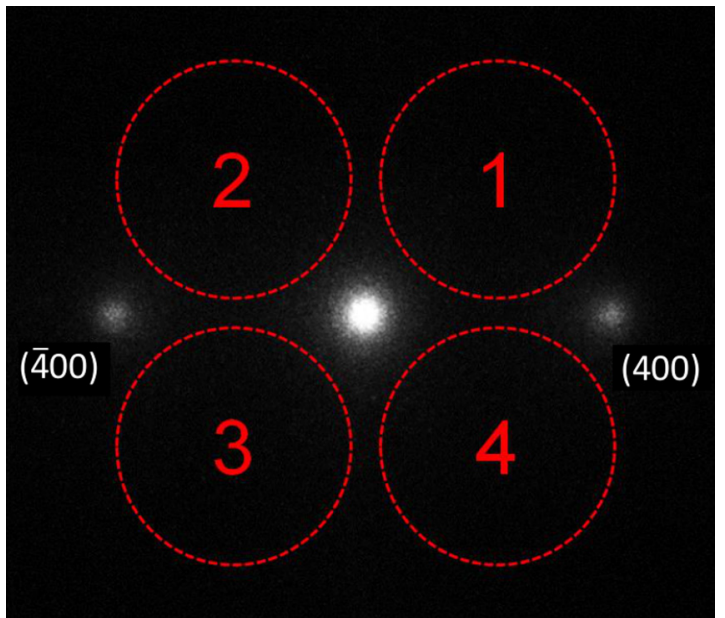

FIG. 4. Experimental electron-diffraction pattern of $\mathrm{NiFe}_{2} \mathrm{O}_{4}$ recorded with $300-\mathrm{keV}$ electrons for a three-beam condition showing a (400) systematic row for a sample of thickness $290 \AA$. The red, dashed circles indicate the positions of detector apertures placed in quadrants $1-4$ in the diffraction plane to record EELS data.

$$
\begin{aligned}
& +2 \sigma_{n}^{2} \Re\left\{\left|C_{\mathbf{0}} \| C_{-\mathbf{G}}\right| \exp \left[i\left(\phi_{-\mathbf{G}}-\phi_{\mathbf{0}}\right)\right]\right. \\
& \left.\times H_{n 0}^{*}\left(\mathbf{q}_{\perp}\right) H_{n 0}\left(\mathbf{q}_{\perp}+\mathbf{G}\right)\right\} \quad(\equiv \text { Term } 1) \\
& +2 \sigma_{n}^{2} \Re\left\{\left|C_{-\mathbf{G}}\right|\left|C_{\mathbf{G}}\right| \exp \left[i\left(\phi_{\mathbf{G}}-\phi_{-\mathbf{G}}\right)\right]\right. \\
& \left.\times H_{n 0}^{*}\left(\mathbf{q}_{\perp}+\mathbf{G}\right) H_{n 0}\left(\mathbf{q}_{\perp}-\mathbf{G}\right)\right\}(\equiv \text { Term } 2) \\
& +2 \sigma_{n}^{2} \Re\left\{\left|C_{\mathbf{0}}\right|\left|C_{\mathbf{G}}\right| \exp \left[i\left(\phi_{\mathbf{G}}-\phi_{\mathbf{0}}\right)\right]\right. \\
& \left.\times H_{n 0}^{*}\left(\mathbf{q}_{\perp}\right) H_{n 0}\left(\mathbf{q}_{\perp}-\mathbf{G}\right)\right\}(\equiv \text { Term } 3) .
\end{aligned}
$$

As before, let us consider a point $\mathbf{q}_{\perp}$ in the first quadrant of the diffraction plane and express this in units of the magnitude of $\mathbf{G}$ using the shorthand notation $\mathbf{q}_{\perp} \equiv(\alpha, \beta)$. Assume $\mathbf{G}$ is on the positive $q_{x}$ axis. Then we can draw up Table I for the arguments that occur in the $H_{n 0}$ 's in each of the terms in Eq. (32) (reading from left to right) and the direction of the corresponding vector cross products in Eq. (28). Vectors used to determine this for quadrants 1 and 4 in the diffraction plane are shown in Fig. 5. We note that in a given quadrant, for a common phase $\phi$ in Eq. (28), there are two terms with a cross product in one direction and one in the opposite sense. An analysis similar to that in Eqs. (27)-(31) for the two-beam case can also be carried out for the three-beam case, but the basic symmetries in the diffraction plane can be deduced from Table I.

To further the discussion, let us now assume that the crystal is centrosymmetric and that we are in the symmetric threebeam orientation. In that case, two nontrivial Bloch states are most strongly excited [38], each with $C_{\mathbf{G}}^{i}=C_{-\mathbf{G}}^{i}$, and it follows from Eq. (22) that $C_{\mathbf{G}}(z)=C_{-\mathbf{G}}(z)$. As a more specific example, let us consider the point $\mathbf{q}_{\perp}=(1 / 2,1 / 2)$ in the first quadrant and the points conjugate to it. Then we can draw up Table II, showing explicitly the arguments that occur in $H_{n 0}$ in each of the terms in Eq. (32) and also their relative contributions to the signal. In assessing the relative contributions of Terms 1 and 3 , account needs to be taken of the magnitude of $q^{\prime 2}$ and $q^{\prime \prime 2}$ in the denominator of the $B_{n 0}^{ \pm}\left(\mathbf{q}_{\perp}^{\prime}, \mathbf{q}_{\perp}^{\prime \prime}\right)$ term in Eq. (28). Term 2 in Eq. (32) does not contribute since $\phi_{\mathbf{G}}-\phi_{-\mathbf{G}}$ is zero for a centrosymmetric crystal.

It is clear from Table I, and the specific example in Table II, that the same signal is obtained for a detector at point $(\alpha, \beta)$ in the first quadrant and at point $(-\alpha,-\beta)$ in the third quadrant. Similarly, a detector at point $(-\alpha, \beta)$ in the second quadrant and $(\alpha,-\beta)$ in the fourth quadrant will yield the same measurement. In contrast, comparing the signal at $(\alpha, \beta)$ to that at $(\alpha,-\beta)$ yields a nonzero EMCD difference signal. There are three other such comparisons that can be made in directions along or perpendicular to the systematic row [39-41].

The experimental results in Fig. 6(a) confirm all these basic symmetries. These results were obtained from a ferrimagnetic $\mathrm{NiFe}_{2} \mathrm{O}_{4}$ single crystal [42] of uniform thickness using an FEI Titan 80-300 transmission electron microscope operated in parallel-beam mode at $300-\mathrm{kV}$ accelerating voltage. The objective lens exerted a magnetic field $\mathbf{B}$ of almost $2 \mathrm{~T}$ on the specimen in the direction antiparallel to the wave vector of the incident beam $\mathbf{k}_{0}$, thus saturating the magnetization $\mathbf{M}$, as indicated in Fig. 6(b). The EELS data, for the Ni $L$ edge, was recorded using a postcolumn Gatan Tridiem system with a 4 mrad collection angle (with detector aperture positions as indicated in Fig. 4). Before forming the difference spectra, background subtraction was carried out using the preedge range from 817 to $847 \mathrm{eV}$, and normalization was done using the postedge sector from 880 to $930 \mathrm{eV}$. The variations of heights of $L_{3}$ are due to statistical noise and asymmetry due to different dynamical diffraction conditions on either side of the systematic row [43].

In Fig. 6(b) we show a simulation of the difference signals at the $L_{2}$ line as a function of the thickness of the specimen. In the simulation, the same parameters were used as in the experiment, and channeling of the electron by the atomic columns both prior to and after the inelastic interactions $[26,44]$ was taken into account in an absorptive multislice model $[26,45]$. The integral involving atomic wave functions implicit in the factors $F_{n 0}^{ \pm}$and $F_{n 0}^{0}$ in Eqs. (14) and (15), respectively, was evaluated using Cowan's program RCN (version 36.2.5) for atomic radial wave functions [23]. The $\mathrm{Ni}^{2+}$ ions in this

\begin{tabular}{|c|c|c|c|c|c|c|c|c|c|c|}
\hline $\mathbf{q}_{\perp}$ & Quadrant & Term 1 & & Dir. & Term 2 & & Dir. & Term 3 & & Dir. \\
\hline$(\alpha, \beta)$ & 1 & $(\alpha, \beta)$ & $(\alpha+1, \beta)$ & $\downarrow$ & $(\alpha+1, \beta)$ & $(\alpha-1, \beta)$ & $\uparrow$ & $(\alpha, \beta)$ & $(\alpha-1, \beta)$ & $\uparrow$ \\
\hline$(-\alpha, \beta)$ & 2 & $(-\alpha, \beta)$ & $(-\alpha+1, \beta)$ & $\downarrow$ & $(-\alpha+1, \beta)$ & $(-\alpha-1, \beta)$ & $\uparrow$ & $(-\alpha, \beta)$ & $(-\alpha-1, \beta)$ & $\uparrow$ \\
\hline$(-\alpha,-\beta)$ & 3 & $(-\alpha,-\beta)$ & $(-\alpha+1,-\beta)$ & $\uparrow$ & $(-\alpha+1,-\beta)$ & $(-\alpha-1,-\beta)$ & $\downarrow$ & $(-\alpha,-\beta)$ & $(-\alpha-1,-\beta)$ & $\downarrow$ \\
\hline$(\alpha,-\beta)$ & 4 & $(\alpha,-\beta)$ & $(\alpha+1,-\beta)$ & $\uparrow$ & $(\alpha+1,-\beta)$ & $(\alpha-1,-\beta)$ & $\downarrow$ & $(\alpha,-\beta)$ & $(\alpha-1,-\beta)$ & $\downarrow$ \\
\hline
\end{tabular}
compound are considered to be magnetized with four unpaired spins of the six occupied $3 d$ states pointing antiparallel to the direction of the incident beam. Based on these considerations,

TABLE I. Arguments of the transition potentials in each of the labeled terms in Eq. (32), indicating the direction of the corresponding cross product in the second term of Eq. (28). Referring to Fig. 5, an $\uparrow$ indicates that the cross product is out of the page and a $\downarrow$ that it is into the page. 


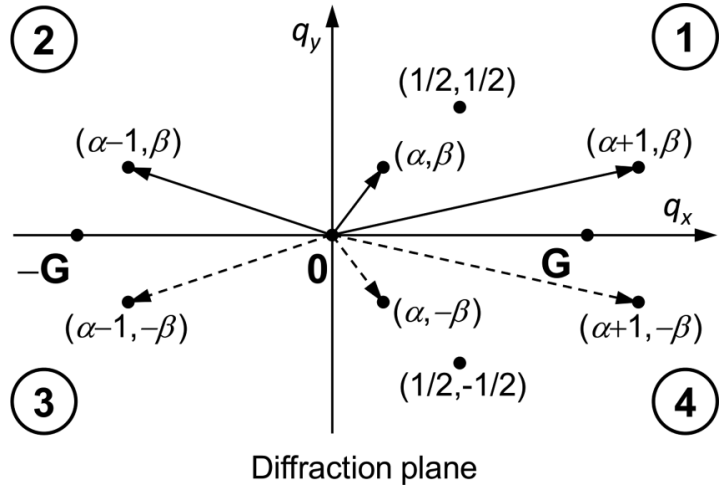

FIG. 5. Three-beam conditions for CTEM showing the various vectors that are pertinent to generic vector $\mathbf{q}_{\perp}=(\alpha, \beta)$ in quadrants 1 (solid lines) and 4 (dashed lines) in Table I. The vectors pertinent to quadrants 2 and 3 are omitted for clarity.

we obtain the signal relative to the incident beam current. This order-of-magnitude estimate shows that for typical beam currents and exposure times a noisy signal is to be expected due to a low number of contributing electrons. Small differences consistent with the asymmetric diffraction conditions already alluded to can be seen. The maximum EMCD difference signal occurs for a thickness of $240 \AA$, a thickness which is slightly smaller than the $290 \AA$ estimated experimentally by the log-ratio method [2]. The diffraction pattern formed by the inelastically scattered electrons and from which the difference signals are formed for the thickness of $240 \AA$ is shown in the inset. Detector apertures of $4 \mathrm{mrad}$ semicollection angle are marked and labeled in the inset. We also note that the thickness at which $\left|C_{\mathbf{G}}\right| /\left|C_{\mathbf{0}}\right|$ [cf. Eq. (22)] is a maximum and thus predicts optimum three-beam conditions is $210 \AA$, slightly lower than the thickness where the difference signal is predicted to be a maximum.

\section{ELECTRON MAGNETIC CIRCULAR DICHROISM IN SCANNING TRANSMISSION ELECTRON MICROSCOPY}

We now discuss EMCD in the context of scanning transmission electron microscopy. In STEM the probe wave function is usually written in the form

$$
\psi_{0}\left(\mathbf{q}_{\perp}\right)=A\left(\mathbf{q}_{\perp}\right) \exp \left[-i \chi\left(\mathbf{q}_{\perp}\right)\right] \exp \left(-2 \pi i \mathbf{q}_{\perp} \cdot \mathbf{R}\right),
$$

where $A\left(\mathbf{q}_{\perp}\right)$ is the aperture function, $\chi\left(\mathbf{q}_{\perp}\right)$ describes the coherent aberrations of the probe, and the position of the probe $\mathbf{R}$ is taken into account by the final phase term. Then Eq. (19) becomes

$$
\begin{aligned}
\left|\psi_{n}\left(\mathbf{q}_{\perp}, z\right)\right|^{2}= & \sigma_{n}^{2} \iint H_{n 0}^{*}\left(\mathbf{q}_{\perp}^{\prime}\right) H_{n 0}\left(\mathbf{q}_{\perp}^{\prime \prime}\right) \\
& \times \exp \left[2 \pi i\left(\mathbf{q}_{\perp}^{\prime}-\mathbf{q}_{\perp}^{\prime \prime}\right) \cdot \mathbf{R}\right] \\
& \times A\left(\mathbf{q}_{\perp}-\mathbf{q}_{\perp}^{\prime}\right) A\left(\mathbf{q}_{\perp}-\mathbf{q}_{\perp}^{\prime \prime}\right) \\
& \times \exp \left[i \chi\left(\mathbf{q}_{\perp}-\mathbf{q}_{\perp}^{\prime}\right)\right] \\
& \times \exp \left[-i \chi\left(\mathbf{q}_{\perp}-\mathbf{q}_{\perp}^{\prime \prime}\right)\right] d \mathbf{q}_{\perp}^{\prime} d \mathbf{q}_{\perp}^{\prime \prime},
\end{aligned}
$$

assuming the probe wave function does not depend on $z$.

For transitions $\Delta m= \pm 1$ this can be written in the form

$$
\begin{aligned}
\left|\psi_{n}^{ \pm}\left(\mathbf{q}_{\perp}, z\right)\right|^{2}= & \sigma_{n}^{2} \iint\left(\cos \phi A_{n 0}^{ \pm} \pm \sin \phi B_{n 0}^{ \pm}\right) \\
& \times A\left(\mathbf{q}_{\perp}-\mathbf{q}_{\perp}^{\prime}\right) A\left(\mathbf{q}_{\perp}-\mathbf{q}_{\perp}^{\prime \prime}\right) d \mathbf{q}_{\perp}^{\prime} d \mathbf{q}_{\perp}^{\prime \prime},
\end{aligned}
$$

where

$$
\phi=2 \pi\left(\mathbf{q}_{\perp}^{\prime}-\mathbf{q}_{\perp}^{\prime \prime}\right) \cdot \mathbf{R}+\chi\left(\mathbf{q}_{\perp}-\mathbf{q}_{\perp}^{\prime}\right)-\chi\left(\mathbf{q}_{\perp}-\mathbf{q}_{\perp}^{\prime \prime}\right) .
$$

In the case of CTEM the phases $\phi$ in Eq. (28) varied as a function of $z$. We note, in particular, that here $\phi$ is also a function of $\mathbf{q}_{\perp}$, probe position $\mathbf{R}$, and, as exploited in Ref. [10], the aberrations in the probe. However, $A_{n 0}^{ \pm}$and $B_{n 0}^{ \pm}$have the same meaning as in Eqs. (20) or (28). In what follows we initially assume a thin specimen, consistent with our assumption that the STEM probe does not depend on $z$.

First, we consider the signal when we scan a STEM probe across a single magnetized atom. In Fig. 7 we show the simulated signal in the diffraction plane, using a $200-\mathrm{keV}$ aberration-free probe with a probe-forming aperture of 25 mrad, from dipole-allowed spin-down to spin-down transitions for $\Delta m=0, \pm 1$ and for different probe positions as indicated in the insets in the rightmost panels of Figs. 7(a) and 7(b). There is an asymmetry in the diffraction signal when the probe is off the exact atomic position. The orientation of the asymmetry is perpendicular to the axis joining the probe and the atom. From these observations we conclude that, in general, there are two possibilities to extract an EMCD difference signal using a spectrometer placed off axis. We can use two different probe positions symmetrically placed with respect to the atom for one off-axis detector position. Alternatively, we can use one probe position and two symmetrically placed off-axis detectors.

\begin{tabular}{|c|c|c|c|c|c|c|c|c|c|c|}
\hline $\mathbf{q}_{\perp}$ & Quadrant & Term 1 & & Dir./Cont. & Term 2 & & Dir./Cont. & Term 3 & & Dir./Cont. \\
\hline$\left(\frac{1}{2}, \frac{1}{2}\right)$ & 1 & $\left(\frac{1}{2}, \frac{1}{2}\right)$ & $\left(\frac{3}{2}, \frac{1}{2}\right)$ & $\downarrow 0.3$ & $\left(\frac{3}{2}, \frac{1}{2}\right)$ & $\left(-\frac{1}{2}, \frac{1}{2}\right)$ & $\uparrow 0$ & $\left(\frac{1}{2}, \frac{1}{2}\right)$ & $\left(-\frac{1}{2}, \frac{1}{2}\right)$ & $\uparrow 0.7$ \\
\hline$\left(-\frac{1}{2}, \frac{1}{2}\right)$ & 2 & $\left(-\frac{1}{2}, \frac{1}{2}\right)$ & $\left(\frac{1}{2}, \frac{1}{2}\right)$ & $\downarrow 0.7$ & $\left(\frac{1}{2}, \frac{1}{2}\right)$ & $\left(-\frac{3}{2}, \frac{1}{2}\right)$ & $\uparrow 0$ & $\left(-\frac{1}{2}, \frac{1}{2}\right)$ & $\left(-\frac{3}{2}, \frac{1}{2}\right)$ & $\uparrow 0.3$ \\
\hline$\left(-\frac{1}{2},-\frac{1}{2}\right)$ & 3 & $\left(-\frac{1}{2},-\frac{1}{2}\right)$ & $\left(\frac{1}{2},-\frac{1}{2}\right)$ & $\uparrow 0.7$ & $\left(\frac{1}{2},-\frac{1}{2}\right)$ & $\left(-\frac{3}{2},-\frac{1}{2}\right)$ & $\downarrow 0$ & $\left(-\frac{1}{2},-\frac{1}{2}\right)$ & $\left(-\frac{3}{2},-\frac{1}{2}\right)$ & $\downarrow 0.3$ \\
\hline$\left(\frac{1}{2},-\frac{1}{2}\right)$ & 4 & $\left(\frac{1}{2},-\frac{1}{2}\right)$ & $\left(\frac{3}{2},-\frac{1}{2}\right)$ & $\uparrow 0.3$ & $\left(\frac{3}{2},-\frac{1}{2}\right)$ & $\left(-\frac{1}{2},-\frac{1}{2}\right)$ & $\downarrow 0$ & $\left(\frac{1}{2},-\frac{1}{2}\right)$ & $\left(-\frac{1}{2},-\frac{1}{2}\right)$ & $\downarrow 0.7$ \\
\hline
\end{tabular}
These possibilities are illustrated by the detector positions indicated in Figs. 7(a) and 7(b). The result in 7(c) shows the signal obtained with two off-axis detectors as indicated in 7(b) while scanning along the horizontal axis across the atom. This also shows that the signal strength depends on the distance of

TABLE II. Arguments of the transition potentials in each of the labeled terms in Eq. (32), indicating the direction of the corresponding cross product in Eq. (28) for the specific case $(\alpha, \beta)=\left(\frac{1}{2}, \frac{1}{2}\right)$. Also shown is the fractional contribution of each term to the signal. Referring to Fig. 5, an $\uparrow$ indicates that the cross product is out of the page and a $\downarrow$ that it is into the page. 

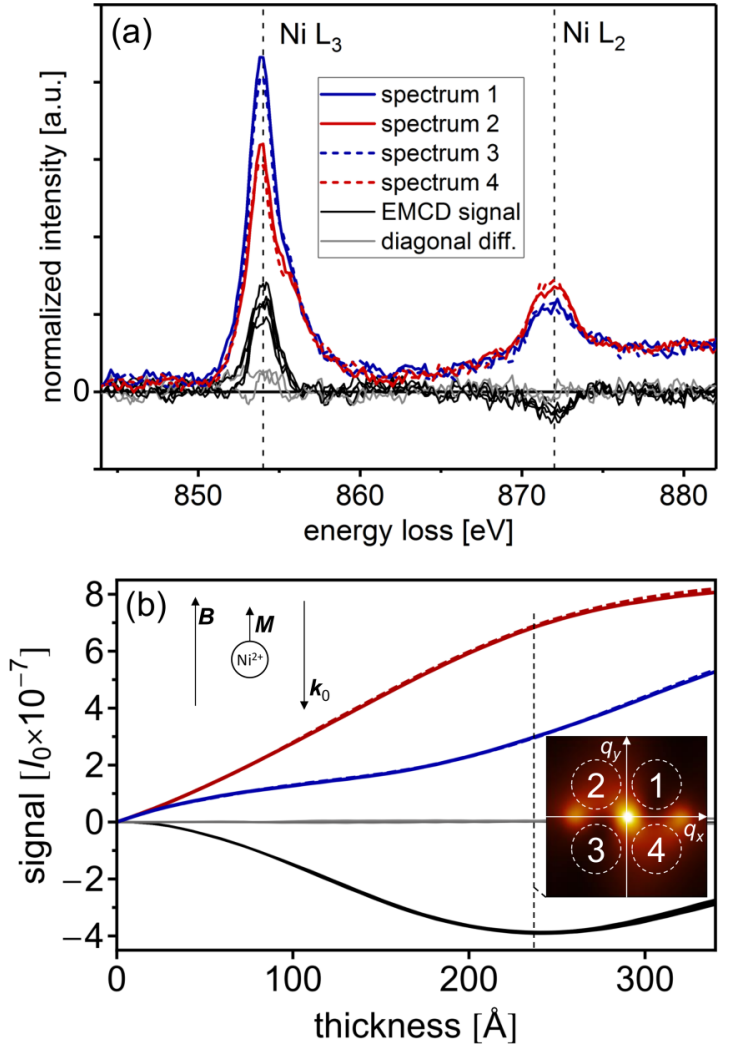

FIG. 6. (a) EELS and difference EMCD spectra for the $\mathrm{Ni} L_{2,3}$ edge in $\mathrm{NiFe}_{2} \mathrm{O}_{4}$ under three-beam (400) systematic row conditions and for the four different detector aperture positions, as indicated in Fig. 4. The four different EMCD signals are obtained by subtracting the spectrum obtained with the detector in the second quadrant from that obtained with the detector in the first quadrant, i.e., spectrum 1 - spectrum 2 (denoted 1-2), and the subtractions 1-4, 3-2, and 3-4. The diagonal differences are obtained from the subtractions 1-3 and 2-4. (b) Simulation of the intensity of the spectra and the difference signals at the $L_{2}$ line as a function of the thickness of the specimen. The difference signal is given relative to the intensity of the incident beam $I_{0}$. In such fractional intensities all equal contributions to the signals before taking the difference (background, nonmagnetic contribution, etc.) cancel in the difference for a given thickness. The diffraction pattern formed by the inelastically scattered electrons and from which the difference signals are obtained for a thickness of $240 \AA$ is shown in the inset. The relative orientations of the external magnetic field $\mathbf{B}$, the magnetization $\mathbf{M}$ of the $\mathrm{Ni}^{2+}$ ions, and the incident-beam wave vector $\mathbf{k}_{0}$ are shown by the arrows inset in (b).

the probe from the atom, in particular, it is localized close to the atomic position.

In Fig. 8 we show simulated STEM EELS images using a 200-keV aberration-free probe, probe-forming semiangle 25 $\mathrm{mrad}$, and for energy-loss electrons of $721 \mathrm{eV}$ (the $\mathrm{Fe} L_{2}$ line) using a detector aperture of $25 \mathrm{mrad}$, and assuming fully spinup polarized atoms. The scan is over the supercell indicated in Fig. 8(a) for bcc $\langle 001\rangle \mathrm{Fe}$ with the unit cell indicated by the dashed lines. Images obtained with an off-axis detector with the entrance aperture centered at $\left(q_{x}, q_{y}\right) / k_{0}=(0,+25) \mathrm{mrad}$ are shown in Fig. 8(b) and for the aperture centered at $\left(q_{x}, q_{y}\right) / k_{0}=$ $(0,-25) \mathrm{mrad}$ in Fig. 8(c). Subtracting the image in Fig. 8(c) from that in Fig. 8(b) yields the EMCD difference map in
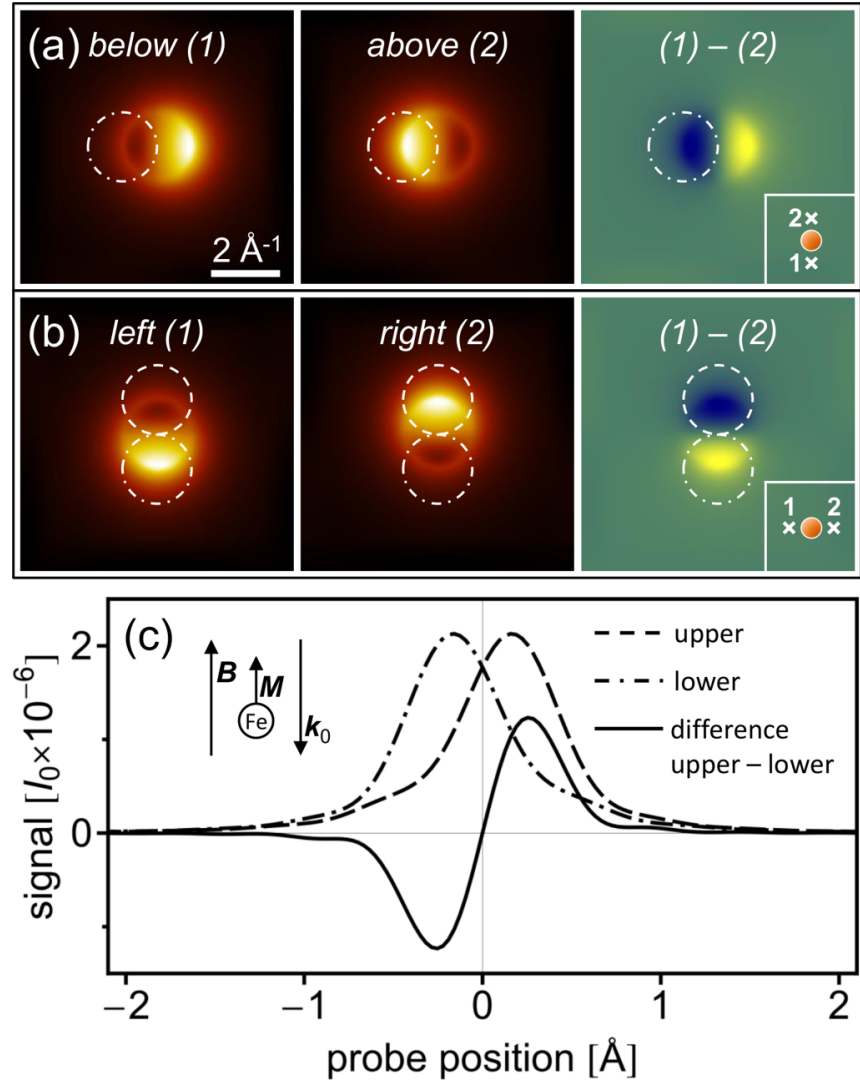

FIG. 7. Simulations of the electron-diffraction signal at 721-eV energy loss for a single spin-up polarized $\mathrm{Fe}$ atom illuminated by a 200-keV aberration corrected STEM probe, considering dipoleallowed transitions. (a) Diffraction patterns with left-to-right asymmetry are obtained when placing the probe above and below the atom, as indicated in the inset on the rightmost panel. (b) Placing the probe left or right of the atom yields a top-to-bottom asymmetry. (c) Horizontal scan of the integrated signals, relative to the incident beam intensity $I_{0}$, into the off-axis detectors indicated in (b) by a dash-dotted circle (upper detector) and a dashed circle (lower detector) with maxima at positions $0.25 \AA$ right and left of the atom, respectively. By taking the difference of these signals, an EMCD signal (solid curve) is obtained, which varies as a function of the probe position. The detectors have a semiangle of $25 \mathrm{mrad}$, the same as that of the probe-forming aperture. The relative orientations of the external magnetic field $\mathbf{B}$, the magnetization $\mathbf{M}$ of the Fe atoms, and the average probe wave vector $\mathbf{k}_{0}$ are shown in part (c) of the figure.

Fig. 8(d) with localized maxima to the left and minima to the right of the atomic positions.

Another possible way to detect EMCD is to use beam shaping, as done in Ref. [10], with the aim of maximizing the EMCD signal. For example, we can construct probes with vortices of opposite chirality. A scan using a vortex probe $[21,22]$ of the form $\exp (i \phi)$ with topological charge +1 is shown in Fig. 8(e) and that with charge -1 in Fig. 8(f) with the detector aperture symmetrically placed on the optical axis. Subtracting the image in Fig. 8(f) from that in Fig. 8(e) results in the EMCD difference signal in Fig. $8(\mathrm{~g})$ that has minima localized at the atomic positions. All plots are on the same relative intensity scale, as indicated by the scale bars. Consistent with the consideration of the localization of the 

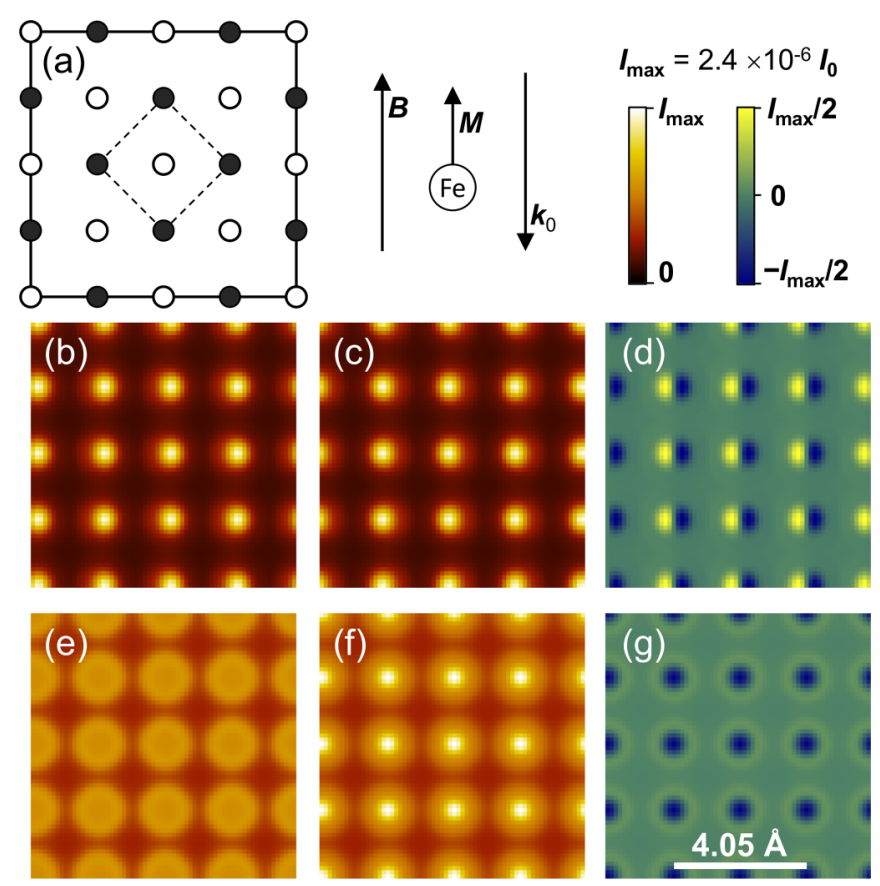

FIG. 8. Simulated 200-kV STEM EELS images using probe and detector semiangles of $25 \mathrm{mrad}$ for an energy loss of $721 \mathrm{eV}$, the Fe $L_{2}$ line, assuming a fully spin-up polarized atom. (a) Supercell used in the simulations for $\langle 001\rangle \mathrm{Fe}$, where the bcc unit cell is marked by dashed lines. Open circles indicate atoms in the upper atomic plane, and filled black circles show atoms in the lower atomic plane. (b) Scan images with an off-axis entrance aperture at $\left(q_{x}, q_{y}\right) / k_{0}=(0,+25) \mathrm{mrad}$ and (c) at $\left(q_{x}, q_{y}\right) / k_{0}=(0,-25)$ mrad yield an EMCD difference image (d) with localized maxima left and minima right of the atomic positions. Scans with vortex probes of topological charge +1 (e) and -1 (f) result in a difference signal $(\mathrm{g})$ with minima localized at the atomic positions. The scans are over the supercell shown in (a). The relative orientations of the external magnetic field $\mathbf{B}$, the magnetization $\mathbf{M}$ of the $\mathrm{Fe}$ atoms, and the average probe wave vector $\mathbf{k}_{0}$ are shown in the upper center part of the figure. The common intensity scales used, defined relative to the intensity of the incident beam $I_{0}$, are shown on the upper right part of the figure, and a spatial scale bar is shown in $(\mathrm{g})$.

transition potentials in Sec. II, Figs. 8(d) and 8(g) clearly show atomically resolved contrast. The width of the signal response from a single atom is likely to be narrower than the interatomic spacing in most materials, thus allowing atomic resolution EMCD.

Thus far we have assumed a thin specimen. However, in thicker samples channeling of the electrons by the atomic columns has an important influence on the measured EMCD signal [13]. In Fig. 9 we show the accumulated difference signal at an energy loss of $721 \mathrm{eV}$ (Fe $L_{2}$ line) for $200-\mathrm{keV}$ electrons incident on bec $\langle 001\rangle \mathrm{Fe}$ for two differently configured probes. The atoms are assumed to be fully spin-up polarized with the magnetization antiparallel to the direction of the incoming beam, and the contribution from allowed $L_{2}$ transitions with $\Delta m=+1$ is larger than from those with $\Delta m=-1$. We take into account the channeling of the electron both before and after the inelastic scattering events and also absorption from the elastic channel due to thermal diffuse scattering.

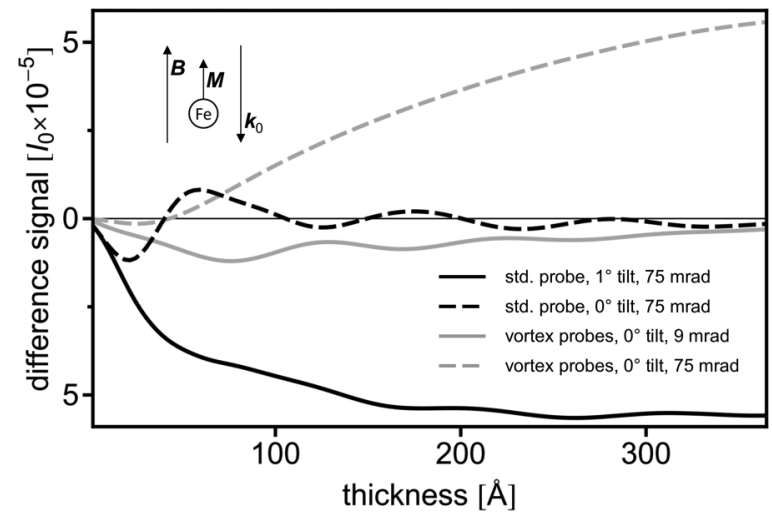

FIG. 9. Accumulated difference signal, relative to the intensity of the incident beam $I_{0}$, for bcc $\langle 001\rangle$ Fe probed with with $200-\mathrm{keV}$ electrons at an energy loss of $721 \mathrm{eV}$ (Fe $L_{2}$ line) assuming fully spinup polarized atoms. The standard STEM probe (denoted std. probe) is positioned $0.25 \AA$ to the left of the atom, an off-axis EELS detector with acceptance semiangle $75 \mathrm{mrad}$ is assumed, and sample tilts of $0^{\circ}$ and $1^{\circ}$ perpendicular to the probe displacement are considered. Vortex probes having topological charges \pm 1 are on an atomic column, and on-axis EELS detectors with collection semiangles of 9 and $75 \mathrm{mrad}$ are assumed. The relative orientations of the external magnetic field $\mathbf{B}$, the magnetization $\mathbf{M}$ of the $\mathrm{Fe}$ atoms, and the average probe wave vector $\mathbf{k}_{0}$ are shown in part (b) of the figure.

First, we show the accumulated difference signal for an aberration-free STEM probe formed using a 25-mrad aperture and placed $0.25 \AA$ to the left of an atom for two off-axis EELS detectors with acceptance semiangles of $75 \mathrm{mrad}$ with the centers at $\left(q_{x}, q_{y}\right) / k_{0}=(0, \pm 75) \mathrm{mrad}$. The STEM probe is to the left of the atom, and the difference was taken by subtracting the signal of the detector in the upper half-plane from that in the lower half-plane. Exactly down the zone axis $\left(0^{\circ}\right.$ tilt $)$ the difference signal is initially negative and then oscillates in sign while decaying with thickness. However, if the sample is tilted by $1^{\circ}$ in the direction perpendicular to the axis joining the probe position and the atomic column, then the difference signal remains negative and, in general, the magnitude increases with thickness. The $1^{\circ}$ tilt was optimal to reduce the pretransition channeling in this example. For less tilt the channeling down columns leads to a cancellation of signal due to an oscillation of the main probe intensity from the left to the right side of the column and vice versa. Tilting by more than $1^{\circ}$ reduces this channeling but also reduces the signal due to cancellations with neighboring columns overlapping in projection. For a tilt of $1^{\circ}$ and sufficiently thin samples, there is still sufficient two-dimensional information to move beyond atomic-plane resolution EMCD (APR-EMCD) [11,13].

Second, we consider the case of vortex probes with topological charges +1 and -1 , also formed with a 25 -mrad probeforming aperture, and using a single on-axis detector with an acceptance semiangle of $75 \mathrm{mrad}$. The probe is on the atomic column with the detector on the optical axis, and the difference is taken by subtracting the signal obtained with a vortex with topological charge -1 from that obtained with topological charge +1 , where the chirality is defined with respect to the incident beam direction. The accumulated difference signal as a function of thickness is significantly influenced by channel- 
ing after the electron has induced an inelastic transition [13]. After initially being negative, at around $40 \AA$ the accumulated difference signal becomes positive. For the vortex probe a smaller detector aperture is needed to avoid the difference signal going positive, as in the result shown for a detector aperture of $9 \mathrm{mrad}$. Unfortunately, the smaller aperture also reduces the difference signal. The standard STEM probe tilted through $1^{\circ}$ with an off-axis detector appears to provide a more favorable scenario to detect the EMCD signal, albeit at the cost of some but not all resolution along the direction of the imposed specimen tilt. It should be noted that spatial incoherence due to a finite source size may affect EMCD mapping using a standard STEM probe and vortex probes in different ways [46].

Although the EMCD signal may have atomic-scale features, it is important to take into account that the degree of localization of the transition potentials can be significant, as discussed in Sec. II, and also that the signal may well have contributions from atoms away from where the probe is positioned because the probe can become distributed over a significant sample volume [47]. So atomic-scale features do not necessarily imply direct atomic resolution information. However, APR-EMCD in STEM, by tilting the specimen away from an exact zone axis orientation, has been theoretically confirmed in Refs. [11] and [13]. For exact zone axis orientation of the specimen, the signal sensitively depends on the probe shape. While in STEM imaging with electron vortex beams the magnetic signal is well localized, for astigmatic probes it is the neighboring atomic columns which contribute the majority of the magnetic signal [48]. Electrons which have previously been thermally scattered (phonon excitation) may also provide a significant component to the signal and from atoms not directly illuminated by the probe, as discussed for EELS and energy-dispersive x-ray elemental mapping [49].

\section{CONCLUSION}

We have introduced an approach based on transition potentials for inelastic scattering to understand the underlying physics of electron magnetic circular dichroism from first principles. This has facilitated an exploration of the localization of the magnetic signal, particularly pertinent for atomic-scale scanning transmission electron microscopy. Furthermore, a direct link is made using an atomic-based model for the electronic structure, in particular, the spin polarization governing the magnetic properties of a material, to the EMCD signal recorded. The transition potential approach lends itself to easy incorporation in multislice electron-diffraction simulations, as has been done here. The multislice approach can efficiently simulate EMCD for nonperiodic systems, and it is possible to incorporate the contribution from thermally scattered electrons, for example, using the quantum excitation of phonons model [50].

Two-beam and three-beam systematic row cases have been discussed in detail for conventional transmission electron microscopy, and the symmetries seen in the theory have been confirmed experimentally. For CTEM we have explicitly assumed centrosymmetric crystals, and consideration of the noncentrosymmetric case is warranted. We have discussed some possible modes of atomic-scale EMCD imaging in STEM, including the use of vortex probes carrying orbital angular momentum. The effects of channeling on the EMCD signal have been addressed.

For STEM, there is much that can still be explored. Significant variables are the dependence on accelerating voltage, probe size and shape, specimen temperature, and the effects of thermal diffuse scattering due to phonon excitation. Quantitative calculations of the EMCD signal, taking into account channeling, enable one to measure magnetization on an absolute scale and allow estimates of experimental parameters, such as acquisition times, in planning atomic-resolution EMCD experiments. Initial and final states, taking into account solidstate effects, should provide a better foundation for further quantitative modeling.

\section{ACKNOWLEDGMENTS}

This work made use of the resources of the National Center for Electron Microscopy in Beijing. P.-L.H. and X.Y.Z. acknowledge financial support from the National Key Research and Development Program (2016YFB0700402), the National Natural Science Foundation of China (51761135131), and the RWTH Aachen University - Tsinghua University Junior Research Fellowship scheme, and also acknowledge helpful input from Z. Q. Wang, J. Zhu, R. Yu, Z. Y. Cheng, S. P. Crane, R. Ramash, and Q. Zhan. This research was supported under the Australian Research Council's Discovery Projects funding scheme (Project DP140102538). L.J.A. acknowledges support by the Alexander von Humboldt Foundation.
[1] P. Schattschneider, S. Rubino, C. Hébert, J. Rusz, J. Kunes, P. Novák, E. Carlino, M. Fabrizioli, G. Panaccione, and G. Rossi, Nature (London) 441, 486 (2006).

[2] R. F. Egerton, Electron Energy-Loss Spectroscopy in the Electron Microscope (Springer Science \& Business Media, New York, 2011).

[3] J. Rusz, O. Eriksson, P. Novák, and P. M. Oppeneer, Phys. Rev. B 76, 060408 (2007).

[4] L. Calmels, F. Houdellier, B. Warot-Fonrose, C. Gatel, M. J. Hÿtch, V. Serin, E. Snoeck, and P. Schattschneider, Phys. Rev. B 76, 060409(R) (2007).

[5] P. Schattschneider, M. Stöger-Pollach, S. Rubino, M. Sperl, C. Hurm, J. Zweck, and J. Rusz, Phys. Rev. B 78, 104413 (2008).
[6] P. Schattschneider, C. Hébert, S. Rubino, M. StögerPollach, J. Rusz, and P. Novák, Ultramicroscopy 108, 433 (2008).

[7] Z. Wang, X. Zhong, R. Yu, Z. Cheng, and J. Zhu, Nat. Commun. 4, 1395 (2013).

[8] J. Rusz, J.-C. Idrobo, and S. Bhowmick, Phys. Rev. Lett. 113, 145501 (2014).

[9] D. Pohl, S. Schneider, J. Rusz, and B. Rellinghaus, Ultramicroscopy 150, 16 (2015).

[10] J.-C. Idrobo, J. Rusz, J. Spiegelberg, M. A. McGuire, C. T. Symons, R. R. Vatsavai, C. Cantoni, and A. R. Lupini, Adv. Struct. Chem. Imaging 2, 5 (2016). 
[11] J. Rusz, S. Muto, J. Spiegelberg, R. Adam, K. Tatsumi, D. E. Bürgler, P. M. Oppeneer, and C. M. Schneider, Nat. Commun. 7, 12672 (2016)

[12] T. Thersleff, J. Rusz, B. Hjörvarsson, and K. Leifer, Phys. Rev. B 94, 134430 (2016).

[13] J. Rusz, J. Spiegelberg, S. Muto, T. Thersleff, M. Ohtsuka, K. Leifer, and P. M. Oppeneer, Phys. Rev. B 95, 174412 (2017).

[14] C. Hébert and P. Schattschneider, Ultramicroscopy 96, 463 (2003).

[15] C. Hébert, P. Schattschneider, S. Rubino, P. Novák, J. Rusz, and M. Stöger-Pollach, Ultramicroscopy 108, 277 (2008).

[16] J. J. Sakurai, Modern Quantum Physics, revised ed. (AddisonWesley, Reading, MA, 1994).

[17] P. Schattschneider, Linear and Chiral Dichroism in the Electron Microscope (Pan Stanford Publishing, Singapore, 2012).

[18] P. Schattschneider, I. Ennen, M. Stöger-Pollach, J. Verbeeck, V. Mauchamp, and M. Jaouen, Ultramicroscopy 110, 1038 (2010).

[19] P. Schattschneider, J. Verbeeck, V. Mauchamp, M. Jaouen, and A.-L. Hamon, Phys. Rev. B 82, 144418 (2010).

[20] P. Schattschneider, B. Schaffer, I. Ennen, and J. Verbeeck, Phys. Rev. B 85, 134422 (2012).

[21] M. Uchida and A. Tonomura, Nature (London) 464, 737 (2010).

[22] J. Verbeeck, H. Tian, and P. Schattschneider, Nature (London) 467, 301 (2010).

[23] R. D. Cowan, The Theory of Atomic Structure and Spectra (University of California Press, Berkeley, CA, 1981).

[24] A. J. D'Alfonso, S. D. Findlay, M. P. Oxley, and L. J. Allen, Ultramicroscopy 108, 677 (2008).

[25] W. Coene and D. Van Dyck, Ultramicroscopy 33, 261 (1990).

[26] C. Dwyer, Ultramicroscopy 104, 141 (2005).

[27] S. D. Findlay, M. P. Oxley, and L. J. Allen, Microsc. Microanal. 14, 48 (2008).

[28] L. J. Allen, A. J. D'Alfonso, and S. D. Findlay, Ultramicroscopy 151, 11 (2015).

[29] D. Song, A. H. Tavabi, Z.-A. Li, A. Kovács, J. Rusz, W. Huang, G. Richter, R. E. Dunin-Borkowski, and J. Zhu, Nat. Commun. 8, 15348 (2017)

[30] L. J. Allen, H. G. Brown, S. D. Findlay, and B. D. Forbes, Microscopy 67 (Suppl 1), i24 (2018).
[31] Z. L. Wang, Elastic and Inelastic Scattering in Electron Diffraction and Imaging (Springer Science \& Business Media, New York, 1995), pp. 185-186.

[32] H. Kohl and H. Rose, Adv. Electron. Electron Phys. 65, 173 (1985).

[33] J. Rusz, S. Rubino, and P. Schattschneider, Phys. Rev. B 75, 214425 (2007).

[34] J. Rusz and J. C. Idrobo, Phys. Rev. B 93, 104420 (2016).

[35] C. J. Humphreys, Rep. Prog. Phys. 42, 1825 (1979).

[36] S. Löffler and P. Schattschneider, Ultramicroscopy 110, 831 (2010).

[37] J. Rusz, P. Novák, S. Rubino, C. Hébert, and P. Schattschneider, Acta Phys. Pol. Series A 113, 599 (2008).

[38] L. J. Allen and C. J. Rossouw, Ultramicroscopy 48, 341 (1993).

[39] H. Lidbaum, J. Rusz, S. Rubino, A. Liebig, B. Hjörvarsson, P. M. Oppeneer, O. Eriksson, and K. Leifer, Ultramicroscopy 110, 1380 (2010).

[40] S. Rubino, P. Schattschneider, J. Rusz, J. Verbeeck, and K. Leifer, J. Phys. D: Appl. Phys. 43, 474005 (2010).

[41] S. Schneider, D. Pohl, S. Löffler, J. Rusz, D. Kasinathan, P. Schattschneider, L. Schultz, and B. Rellinghaus, Ultramicroscopy 171, 186 (2016).

[42] H. Perron, T. Mellier, C. Domain, J. Roques, E. Simoni, R. Drot, and H. Catalette, J. Phys.: Condens. Matter 19, 346219 (2007).

[43] D. Song, Z. Wang, and J. Zhu, Ultramicroscopy 148, 42 (2015).

[44] C. Dwyer, S. D. Findlay, and L. J. Allen, Phys. Rev. B 77, 184107 (2008).

[45] L. J. Allen, S. D. Findlay, M. P. Oxley, and C. J. Rossouw, Ultramicroscopy 96, 47 (2003).

[46] D. T. Nguyen, S. D. Findlay, and J. Etheridge, Ultramicroscopy 146, 6 (2014)

[47] D. T. Nguyen, S. D. Findlay, and J. Etheridge, Ultramicroscopy 184, 143 (2018).

[48] D. Negi, J.-C. Idrobo, and J. Rusz, Sci. Rep. 8, 4019 (2018).

[49] B. D. Forbes, A. J. D’Alfonso, R. E. A. Williams, R. Srinivasan, H. L. Fraser, D. W. McComb, B. Freitag, D. O. Klenov, and L. J. Allen, Phys. Rev. B 86, 024108 (2012).

[50] B. D. Forbes, A. V. Martin, S. D. Findlay, A. J. D'Alfonso, and L. J. Allen, Phys. Rev. B 82, 104103 (2010). 